\title{
Image Retrieval: Color and Texture Combining Based on Query-Image
}

\author{
Ilya Markov ${ }^{1}$ and Natalia Vassilieva ${ }^{2}$ \\ ${ }^{1}$ Saint-Petersburg State University, Russia \\ ilya.markov@gmail.com \\ ${ }^{2}$ HP Labs, Saint-Petersburg, Russia \\ nvassilieva@hp.com
}

\begin{abstract}
It is a common way to process different image features independently in order to measure similarity between images. Color and texture are the common ones to use for searching in natural images. In [10] a technique to combine color and texture features based on a particular query-image in order to improve retrieval efficiency was proposed. Weighted linear combination of color and texture metrics was considered as a mixed-metrics. In this paper the mixed-metrics with different weights are compared to pure color and texture metrics and widely used CombMNZ data fusion algorithm. Experiments show that proposed metrics outperform CombMNZ method in some cases, and have close results in others.
\end{abstract}

Keywords: Content-Based Image Retrieval, Mixed-Metrics, Data Fusion.

\section{Introduction}

Color and texture are the common features which are used for searching in natural images with heterogeneous content. Heterogeneous content means that considered image collection has no common subject or properties. In case collection has one common theme (for example, facial collection, collection of finger prints, medical shots collection), it might be possible to use some particular set of features adjusted to the theme, which might be more effective.

Common approach in image retrieval is to measure similarity between images based on different features independently and than combine these results together in order to get final result. While there are a lot of studies on different low-level image features analysis [12, not so many researches consider a task of combining various similarity measures based on different image features.

In image retrieval linear combination is commonly used to combine multiple searchers in order to get the final result [12 5]. The main reason for that is the simplicity of the algorithm. Text retrieval research domain has longer history and more experience. It is possible to borrow some methods from that area and apply them successfully to image retrieval. CombMNZ [4] is considered to be one of the best data fusion algorithms for combining multiple searches in text retrieval

\footnotetext{
^ This work was partially supported by RFBR (grant 07-07-00268a).
} 
[11. In [17] we showed that it can be used in image retrieval also: CombMNZ outperforms linear combination in most cases.

In [10] we proposed a technique to combine color and texture metrics taking into account a particular query-image without interaction between system and human. Weighed linear combination of color and texture metrics (mixed-metrics) is considered as a fusion function. Our approach is based on the hypothesis, proposed and proved in [10], that there are optimal weights to combine color and texture metrics for every query-image and these weights are unique for a given query. By using these optimal weights one can improve retrieval results. We showed that it is always possible to mark out the best mixed-metrics for every group of similar images (and thus for every query-image). In other words, we proposed adaptive fusion algorithm without using relevance feedback.

It is possible that one can obtain better results by utilizing relevance feedback algorithms, which are sometimes used to obtain optimal coefficients for every particular query to combine different similarity measures [1]. But, on one hand, not all image retrieval systems have relevance feedback implementation and, on the other, our method can improve even those which have. When relevance feedback is used, improvement can be achieved starting from the second iteration. Our method can be used to get more precise retrieval results on the first iteration, when no feedback from user is yet available.

In this study we continue to investigate the approach described above and compare it to CombMNZ 4 algorithm. Experimental results show that mixedmetrics outperform CombMNZ method in some cases, and have close results in others. In average mixed-metrics slightly outperform CombNMZ algorithm: average precision of mixed-metrics search is $42.76 \%$ and the same for CombMNZ is $39.68 \%$.

\section{Related Works}

Many researchers showed that it is necessary to combine various features for effective image retrieval. At the same time not enough attention is paid to the particular fusion methods. A number of works dedicated to similarity measures combining for image retrieval task is relatively small.

In [3] authors examine an application of a fuzzy logic approach to the fusion of image features. While it is a promising technique, there is no similarity measure proposed for observed fused feature and no experimental or other results are shown that can prove an efficiency of this approach.

Common solution is to fuse similarity measures calculated based on different features but not the features themselves. Linear combination of multiple similarity measures is usually treated as an aggregate measure (in [5] for instance).

Common data fusion algorithms like CombSUM, CombMNZ [4] and others 817] are widely used in text retrieval. The same algorithms can be applied to image retrieval domain. 
CombMNZ is considered to be one of the best data fusion algorithms. It performs as follows. Element in the result ranked-list gets rank equaled to the sum of all its ranks in fused lists divided by the number of lists in which this element exists with non-zero rank:

$$
\begin{gathered}
\operatorname{rank}_{\text {result }}(o b j)=\sum_{\text {fused lists }} \operatorname{rank}_{\text {list }}(o b j) \cdot n z, \quad \forall \text { obj } \in \text { image collection, } \\
\text { where } n z=\sum_{\text {fused lists }}\left(\operatorname{rank}_{\text {list }}(o b j)=0 ? 0: 1\right) .
\end{gathered}
$$

This algorithm is simple to use and outperforms other data fusion methods [7].

In [17] we proposed our own data fusion method "Weighted Total with Gravitation Function" (WTGF) and compared it to CombMNZ, applied to the image retrieval domain. WTGF function satisfies various criteria like symmetry, monotonicity and so called "cone rules". Experimental results showed that WTGF outperforms CombMNZ in case there are multiple inputs of non-equal reliability (we can trust to one input more than to others) and inputs do not overlap much. In case information about element ranks is not trusted (all inputs have the same reliability) and inputs overlap a lot, CombMNZ outperforms WTGF.

Combination of search results obtained by using color and texture features is the second case. Therefore we compare mixed-metrics to CombMNZ algorithm in this work.

\section{Mixed-Metrics}

In 10] we proposed a technique to combine image similarity measures which takes into account a particular query-image. We introduced mixed-metrics obtained from color and texture metrics ( $C$ and $T$ respectively) by using their weighted linear combination $a \cdot C+(1-a) \cdot T$, where $a$ is a varying coefficient which depends on a query-image.

We stated and proved the hypothesis that optimal value of $a$ is the same for similar query-images. It means that in order to perform a search by using mixedmetrics one should go through the following steps. Entire image collection on which a search to be performed should be prepared as follows. Get some relatively small training set of images representing the whole collection and divide it into groups of similar images. Than calculate an average precision for every group for different values of $a$ (varying from 0 to 1 with predefined step) applied to mixed-metrics. An average precision for a group is calculated based on retrievals when group's images are used as queries. Finally, select optimal coefficient $a$ based on precision values and calculate "average" features for each group. One of the main ideas here is that this preparation should be done only once for the collection. It is also possible to use the same training set for several collections in case it represents all of them well.

To perform a search itself, one should classify query-image to one of the groups of the training set. After classification a search can be performed by using mixedmetrics of the group which query-image belongs to. 


\section{Color and Texture Features Selection}

We use moment based color distribution features and color metrics from [15]. This approach is more robust in matching colors than the one based on classic color histograms [14. In 15] color is represented with its mean for every channel and the covariance matrix of the channels' distributions. Minimal amount of spatial information is encoded into color index: each image is divided into five partially overlapping fuzzy regions. Feature vector is calculated for every region. Weighted Manhattan distance is used as a similarity function.

While ICA filters are more natural comparing to Gabor filters, we use convolutions of image with ICA filters as a texture feature and Kullback-Leibler divergence as a texture metrics 2. Texture features built by using Gabor filters are one of the most popular approaches for texture. It was shown that Gabor-based features outperform other texture features in a query-by-example approach to image retrieval [9]6. In [13] it was shown that ICA-filters perform better in classification task, therefore we can assume that they are better in retrieval task too.

\section{$5 \quad$ Experiment}

Experimental image database consists of 650 images from Corel Photo Set collection. It is divided into 9 groups based on images content by 2 experts. Result groups are: City, Clouds, Coastal landscapes, Contemporary buildings, Fields, Lakes, People, Rocks and Trees. This set of images can be considered as a training set for some larger collection.

For every image in the database color and texture features are extracted and for every pair of images color and texture distances are computed. Distance values are normalized according to the following rule:

$$
\text { distance }_{\text {result }}(\text { image })=(\text { distance }(\text { image })-\text { Average }) / \text { Deviation } \text {. }
$$

Therefore distributions of color and texture distances have the same Average and Deviation.

CombMNZ and several mixed-metrics are evaluated in our experiment. Participated mixed-metrics are: $a \cdot C+(1-a) \cdot T$, where $a$ varies from 0 to 1 with 0.1 step. Mixed-metrics with $a=0$ is a pure texture metrics and with $a=1$ is a pure color one.

To estimate retrieval efficiency we use average precision at $\mathrm{N}$ measure - a common one in information retrieval. Precision at $\mathrm{N}$ is a percentage of relevant objects among first $\mathrm{N}$ retrieved. To obtain average precision at $\mathrm{N}$ for all fusion methods the following procedure is performed. Every image in the database is used as a query. One search per every query and fusion method is run and precision at $\mathrm{N}$ for each run is calculated $(\mathrm{N}=1 . .30)$. Images from the same group as the query-image are treated as relevant, while others are not. Average precision at $\mathrm{N}$ is calculated for every group of similar images and every fusion method. 


\section{Results Analysis}

Summary results are shown in table 1 for the following search methods: pure color- and texture-based (ColorMoments and ICA respectively), CombMNZ and best mixed-metrics-based (in accordance with the particular group). For every group average precision at $\mathrm{N}$ is calculated for every method for different $\mathrm{N}$.

Table 1. Best mixed-metrics precision compared to other metrics precisions for every group

\begin{tabular}{|c|c|c|c|c|c|c|c|}
\hline Group & Search Algorithm & $\mathrm{N}=5$ & \multicolumn{4}{|c|}{ Average precision at N (\%) } & $\mathrm{N}=30$ \\
\hline \multirow{4}{*}{ City } & Color Moments & 24 & 24 & 21 & 19 & 19 & 18 \\
\hline & ICA & 31 & 27 & 26 & 26 & 23 & 22 \\
\hline & CombMNZ & 39 & 32 & 27 & 24 & 22 & 20 \\
\hline & Mixed-Metrics (0.2) & 44 & 38 & 35 & 33 & 30 & 29 \\
\hline \multirow{4}{*}{ Clouds } & Color Moments & 83 & 81 & 80 & 77 & 76 & 74 \\
\hline & $\mathrm{ICA}$ & 68 & 62 & 57 & 52 & 49 & 47 \\
\hline & CombMNZ & 82 & 80 & 79 & 78 & 77 & 75 \\
\hline & Mixed-Metrics (0.5) & 86 & 81 & 79 & 78 & 76 & 75 \\
\hline \multirow{4}{*}{ Coastal Landscapes } & Color Moments & 36 & 34 & 33 & 32 & 31 & 31 \\
\hline & $\mathrm{ICA}$ & 20 & 17 & 16 & 16 & 15 & 15 \\
\hline & CombMNZ & 36 & 33 & 33 & 33 & 32 & 31 \\
\hline & Mixed-Metrics (0.8) & 36 & 34 & 33 & 31 & 32 & 30 \\
\hline \multirow{4}{*}{ Contemp. Buildings } & Color Moments & 30 & 28 & 28 & 28 & 27 & 27 \\
\hline & ICA & 22 & 21 & 21 & 19 & 19 & 18 \\
\hline & CombMNZ & 30 & 29 & 31 & 30 & 30 & 30 \\
\hline & Mixed-Metrics (0.2) & 29 & 32 & 32 & 33 & 32 & 32 \\
\hline \multirow{4}{*}{ Fields } & Color Moments & 51 & 50 & 48 & 45 & $\overline{44}$ & $\overline{43}$ \\
\hline & $\mathrm{ICA}$ & 38 & 35 & 33 & 32 & 32 & 31 \\
\hline & CombMNZ & 49 & 45 & 44 & 44 & 44 & 44 \\
\hline & Mixed-Metrics (1.0) & 51 & 50 & 48 & 45 & 44 & 43 \\
\hline \multirow{4}{*}{ Lakes } & Color Moments & 45 & 43 & 43 & 42 & 41 & 40 \\
\hline & $\mathrm{ICA}$ & 24 & 23 & 23 & 22 & 23 & 23 \\
\hline & CombMNZ & 41 & 41 & 39 & 39 & 38 & 37 \\
\hline & Mixed-Metrics (0.8) & 46 & 46 & 45 & 43 & 42 & 41 \\
\hline \multirow{4}{*}{ People } & Color Moments & 32 & 33 & 30 & 28 & 27 & - \\
\hline & $\mathrm{ICA}$ & 26 & 20 & 20 & 18 & 17 & - \\
\hline & CombMNZ & 40 & 34 & 31 & 29 & 27 & - \\
\hline & Mixed-Metrics (0.7) & 42 & 39 & 39 & 36 & 35 & - \\
\hline \multirow{4}{*}{ Rocks } & Color Moments & 42 & 36 & 34 & 32 & - & - \\
\hline & $\mathrm{ICA}$ & 6 & 5 & 4 & 5 & - & - \\
\hline & CombMNZ & 46 & 36 & 33 & 28 & - & - \\
\hline & Mixed-Metrics (1.0) & 42 & 36 & 34 & 32 & - & - \\
\hline \multirow{4}{*}{ Trees } & Color Moments & 35 & 33 & 32 & 32 & 32 & 32 \\
\hline & $\mathrm{ICA}$ & 27 & 24 & 23 & 23 & 21 & 21 \\
\hline & CombMNZ & 43 & 40 & 35 & 34 & 32 & 32 \\
\hline & Mixed-Metrics (0.2) & 45 & 44 & 42 & 40 & 40 & 39 \\
\hline
\end{tabular}




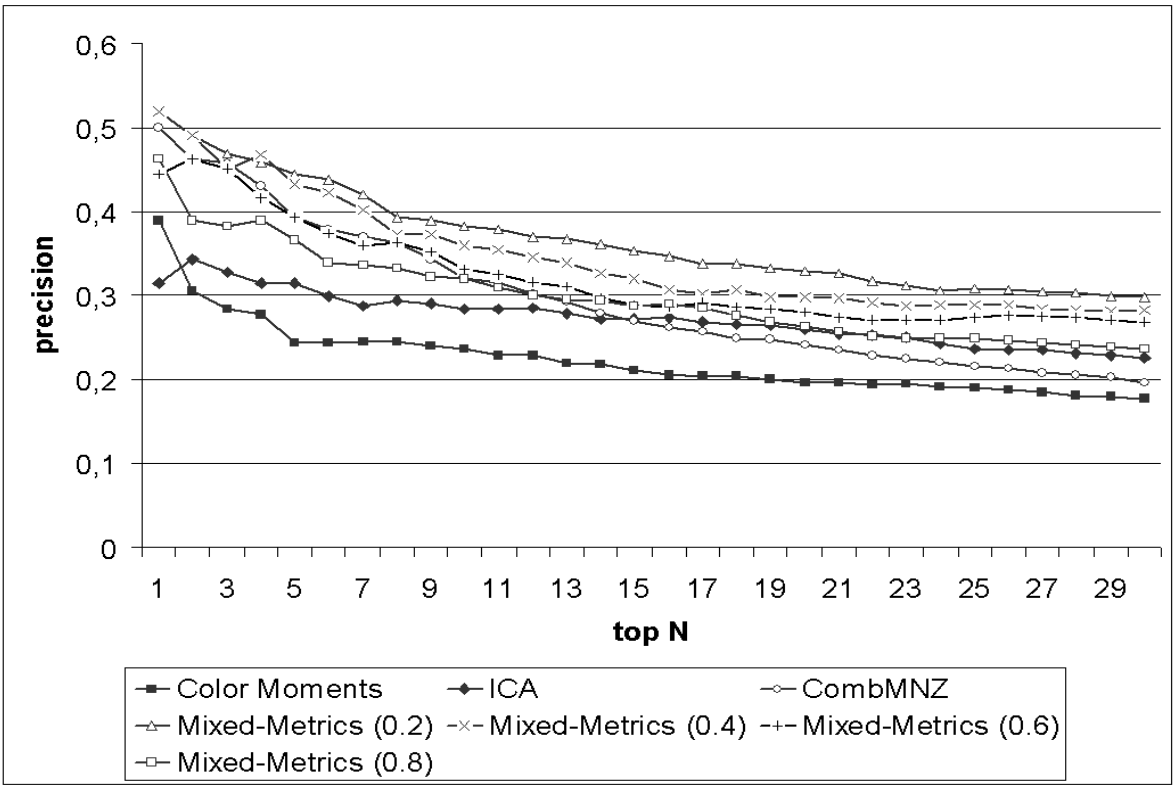

Fig. 1. Precision/Top N dependencies for different searches for group "City"

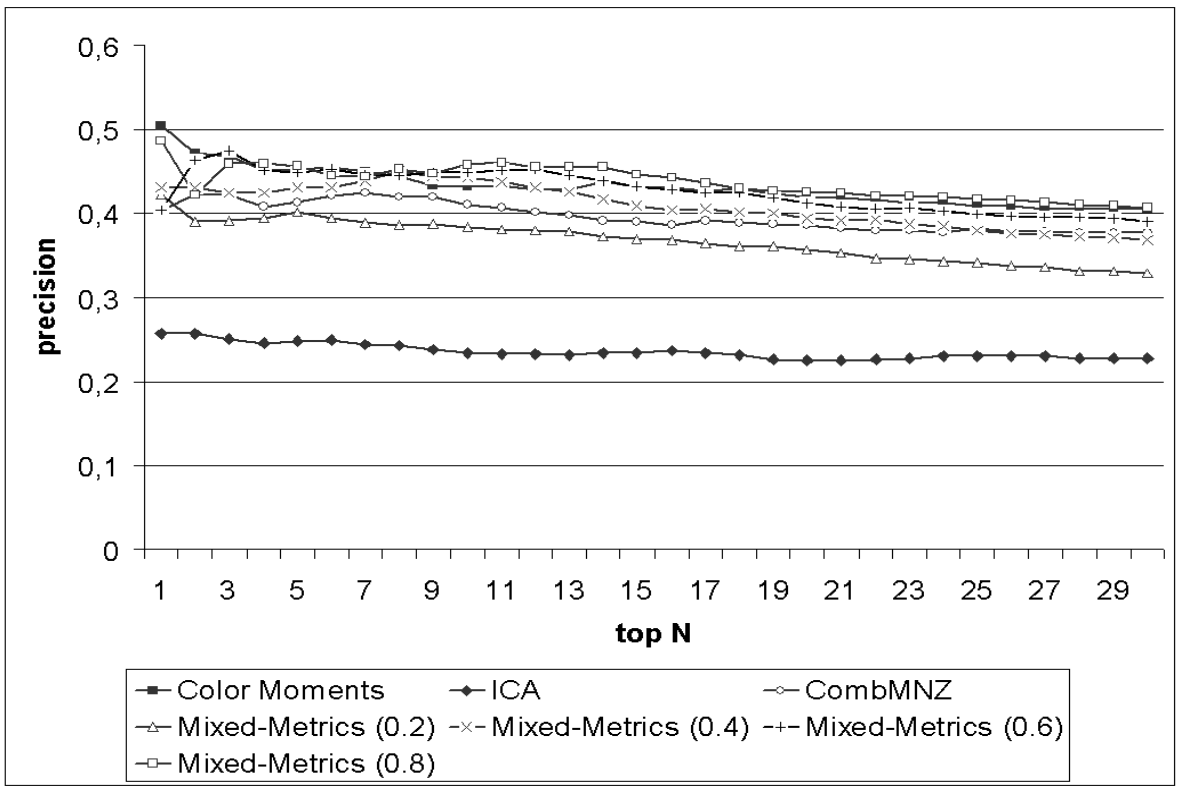

Fig. 2. Precision/Top N dependencies for different searches for group "Lakes" 


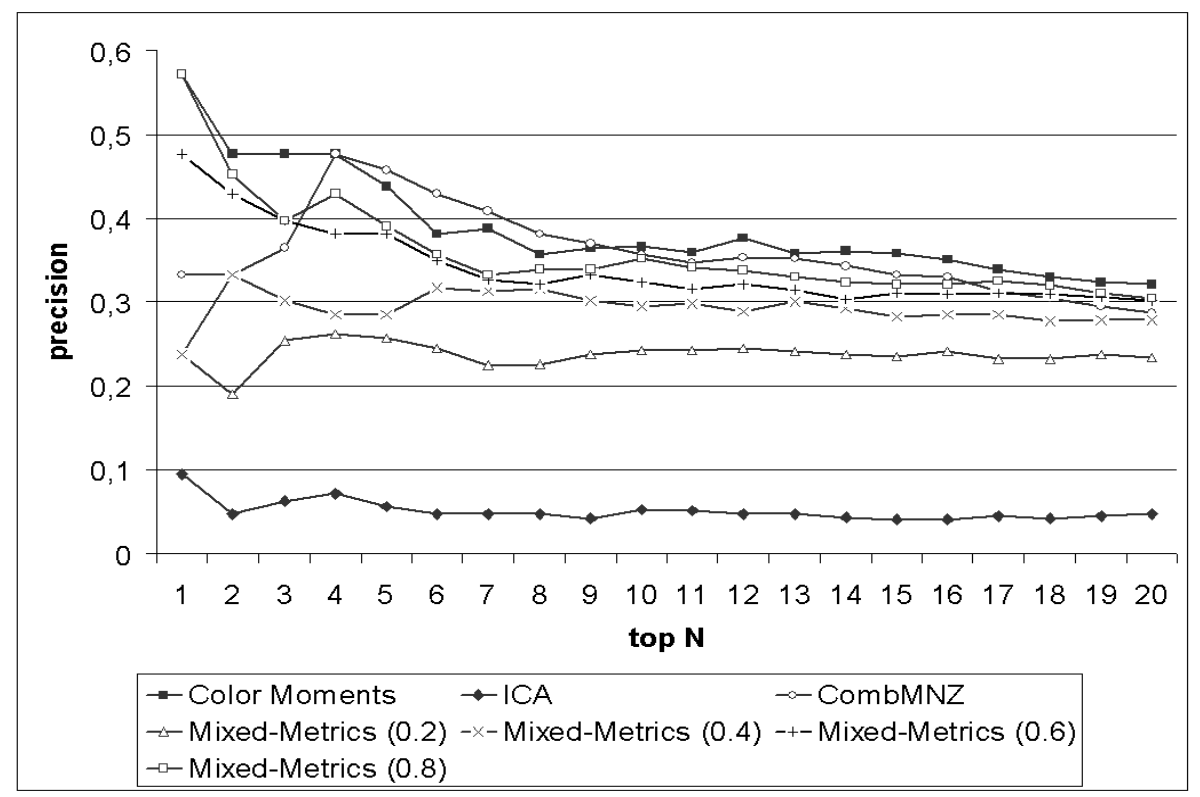

Fig. 3. Precision/Top N dependencies for different searches for group "Rocks"

Results show that in most cases a search with best group mixed-metrics has greater precision compared to runs with CombMNZ. For other groups ("Clouds", "Coastal landscapes" and "Rocks") results of both methods are very close to each other. Average precision of mixed-metrics search among all images of the database is $42.76 \%$ and the same for CombMNZ is $39.68 \%$.

Let us discuss results for some groups in detail. Result charts for these groups ("City", "Lakes" and "Rocks") are shown on fig. 1, 2 and 3 respectively.

The precision/top N dependencies for different searches for group "City" are shown on fig. 1. Texture search gives more precise result than color one and therefore texture feature is more important for this group. Search with $0.2 \cdot C+$ $0.8 \cdot T$ mixed-metrics gives the best result and also proves the above statement. Results for other mixed-metrics searches show that precision decreases when mixed-metrics moves from texture to color. Precision of CombMNZ algorithm decreases faster than precision of color and texture searches. For fist 10 positions it loses only $5 \%$ compared to $0.2 \cdot C+0.8 \cdot T$ mixed-metrics. And for 30 th position it loses $10 \%$.

The inverse situation can be seen on fig. 2 for group "Lakes". Color feature is more important here and precision decreases when mixed-metrics moves from color to texture. Best result is obtained by using $0.8 \cdot C+0.2 \cdot T$ mixed-metrics.

Group "Rocks" on fig. 3 is the case when search with pure color metrics and CombMNZ algorithm give more precise results. Pure color metrics can be treated as $1.0 \cdot C+0.0 \cdot T$ mixed-metrics here. 


\section{Conclusions and Further Work}

Experiments show that mixed-metrics improve retrieval results compared to pure color and pure texture metrics. This result proves common observation that combined search with several features gives better results than individual searchers. Moreover mixed-metrics outperform CombMNZ data fusion algorithm in some cases and give close results in others.

As it was mentioned in section 3 in order to perform retrieval using the optimal mixed-metrics a query-image should be classified to one of the groups established in training set. Therefore the next step of our research is to provide an efficient and effective classification algorithm for this task. While classification should be performed in real time during retrieval process it should be as fast as possible, therefore it should be simple enough and involve just a few computations. For this reason, many well-known classification algorithms cannot be used in our environment.

Possible solution here is to compute common color and texture features for obtained groups. Then the classification can be done through comparing queryimage features to groups' common features. It is important that groups' common features should differ one from another. Otherwise it is impossible to perform classification task and obtain optimal mixed-metrics for any query-image.

Acknowledgments. This work was partially supported by RFBR (grant 0707-00268a).

\section{References}

1. Aksoy, S., Haralick, R.M., Cheikh, F.A., Gabbouj, M.: A Weighted Distance Approach to Relevance Feedback. In: 15th International Conference on Pattern Recognition, vol. 4, pp. 812-815 (2000)

2. Borgne, H., Guerin-Dugue, A., Antoniadis, A.: Representation of images for classification with independent features. Pattern Recognition Letters 25, 141-154 (2004)

3. Deer, P.J., Eklund, P.W.: On the Fusion of Image Features, http://citeseer.ist.psu.edu/162546.html

4. Fox, E.A., Shaw, J.A.: Combination of multiple searches. TREC 2, 243-249 (1994)

5. Guerin-Dugue, A., Ayache, S., Berrut, C.: Image retrieval: a first step for a human centered approach. In: Joint Conference of ICI, CSP and PRCM, vol. 1, pp. $21-$ 25 (2003)

6. Howarth, P., Rueger, S.: Robust texture features for still image retrieval. In: IEE Proc. of Vision, Image and Signal Processing, vol. 152(6), pp. 868-874 (2005)

7. Lee, J.H.: Analyses of Multiple Evidence Combination. In: ACM-SIGIR, USA, pp. 267-276 (1997)

8. Lilis, D., Toolan, F., Mur, A., Peng, L., Colier, R., Dunnion, J.: Probability-Based Fusion of Information Retrieval Result Sets. J. Artif. Intell. Rev. 25(1-2), 179-191 (2006)

9. Manjunath, B.S., Ma, W.Y.: Texture features for browsing and retrieval of image data. IEEE Transactions on Pattern Analysis and Machine Intelligence 18(8), 837842 (1996) 
10. Markov, I., Vassilieva, N., Yaremchuk, A.: Image retrieval. Optimal weights for color and texture features combining based on query object. In: Proc. of RCDL, Russia, pp. 195-200 (2007)

11. Montague, M., Aslam, J.A.: Relevance Score Normalization for Metasearch. In: ACM Conference on Information and Knowledge Management, pp. 427-433 (2001)

12. Rui, Y., Huang, T.S., Chang, S.-F.: Image Retrieval: Past, Present and Future. In: International Symposium on Multimedia Information Processing (1997)

13. Snitkowska, E., Kasprzak, W.: Independent Component Analysis of Textures in Angiography Images. Computational Imaging and Vision 32, 367-372 (2006)

14. Stricker, M., Dimai, A.: Color Indexing with Weak Spatial Constraints. In: Storage and Retrieval for Image and Video Databases (SPIE), pp. 26-40 (1996)

15. Stricker, M., Dimai, A.: Spectral Covariance and Fuzzy Regions for Image Indexing. In: Machine Vision and Applications, vol. 10, pp. 66-73 (1997)

16. Swain, M., Ballard, D.: Color Indexing. International Journal of Computer Vision 7(1), 11-32 (1991)

17. Vassilieva, N., Dolnik, A., Markov, I.: Image retrieval. Fusion of the result sets retrieved by using different image characteristics. Internet-mathematics Collection, 46-55 (2007) 